\title{
Multidrug resistant extrapulmonary tuberculosis - three case reports and review of literature
}

\author{
Neeru Mittal ${ }^{* *}$ and Punit Bansal ${ }^{2}$ \\ *Correspondence: drneerub@gmail.com

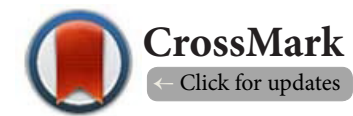 \\ 'Department of Chest Medicine, Christian Medical College, Ludhiana, Punjab, India. \\ ${ }^{2}$ RG Stone and Super Speciality Hospital, Ludhiana, Punjab, India.
}

\begin{abstract}
We present three cases of extra pulmonary multidrug-resistant tuberculosis and treated with second line drugs according to culture susceptibility pattern. On completion of therapy, patents showed good clinical response. These cases highlight the observation that even extra-pulmonary multidrug-resistant tuberculosis in on the rise and can be successfully treated with currently available second line drugs. All the Physicians should be cautious while dealing with recurrent cases of tubercular lymphadenitis.
\end{abstract}

Keywords: Multi drug, resistant, tuberculosis, therapy

\section{Introduction}

Multi-drug resistant extrapulmonary tuberculosis is an uncommon form of the disease, but it seems that by increasing the number of drug resistant tuberculosis around the world, the number of cases of multi-drug resistant tuberculosis with extrapulmonary presentation also is going to rise. By increasing the number of drug resistant tuberculosis patients around the world, appropriate diagnosis and treatment of different presentations of the disease need a special attention [1]. Diagnosis is difficult often requiring biopsy for several times. Treatment monitoring is more complex due to peculiar behaviour of TB lymph nodes. Along with rise of multi-drug resistance (MDR), drug-resistant TB lymphadenitis cases are also on the rise [2]. We present 3 cases that highlights the observation that even extra-pulmonary primary multidrugresistant tuberculosis can be successfully treated with currently available second line drugs.

\section{Case presentation}

Case 1

A 23 year old nursing student presented with swelling in the right side of neck for 15 months. She had low grade fever and had loss of weight of 5-7 kgs in last 2 months. FNAC was done in a private centre that suggested tuberculosis and then she underwent lymph node excision biopsy and pathology suggested tubercular lymphadenitis. She was started on DOTS category 1 ATT. Her fever and symptoms subsided but her lymph nodes increased in number and size within one month of starting ATT.

Then she was started on daily regimen of ATT but her symptoms did not improve. She had fever and pus draining sinus for which she was referred to us. Again FNAC was done which suggested Tuberculosis. Pus was sent for BACTEC culture for tuberculosis and five drug sensitivity test. Pus was resistant to Isoniazid and rifampicin. Her blood investigations were normal. She was negative for HIV. ESR was 80 . Chest $X$ ray showed increased bronchovasular markings. Mantoux test showed induration of $15^{*} 12 \mathrm{~mm}$ Her USG neck showed illdefined heterogenous collection of 7-10 cc along right sternocleidomastoid.

She was started on $2^{\text {nd }}$ line ATT, Kanamycin $0.75 \mathrm{~g}$, levoflox 750 $\mathrm{mg}$, Cycloserine $750 \mathrm{mg}$, ehionamide 750, PAS powder $10 \mathrm{gm}$ and ehambutol $1.2 \mathrm{~g}$, Pyrazimide $1.5 \mathrm{~g}$. Her symtoms improved after 3 months and lymph node size and pus drainage stopped after 3 months. Patient completed 24 months of treatment and she had no recurrence till date.

Case 2

A 35 year muslim man presented with swelling in the left side of neck of 2 years duration. He complained of loss of weight and loss of appetite of last 10 months. He had first episode of tubercular cervical lymphadenitis 2 years back for which he was treated with category 1 . He remained well for three months and then again developed increase in size of lymph node and new nodes. He was treated with category 2 but lymph nodes did not resolve completely. Then again he developed increase 
Mittal et al. Internal Medicine Inside 2014,

in size of lymph nodes and appearance of new nodes after 4 months. The swelling was tender and had discharging sinuses.

He had Hemoglobin of 12 and ESR was 100. Rest of blood investigations were normal. Chest $X$ ray did not reveal any gross abnormality Mantoux test was positive FNAC was done which suggested Tuberculosis. Pus was sent for BACTEC culture for Mycobacterium tuberculosis and five drug sensitivity test. Pus was resistant to Isoniazid and rifampicin and pyrazinamide. He was negative for HIV. She was started on $2^{\text {nd }}$ line ATT, Kanamycin 0.75 g, levoflox 750 mg, Cycloserine 750 mg, ehionamide 750, PAS powder $10 \mathrm{gm}$ and ethambutol 1.2 g. His symptoms improved after 5 months and lymph node size and pus drainage stopped after 3 months. Patient has completed 10 months of treatment and has still no recurrence.

\section{Case 3}

A 30-year, non-smoker male was admitted with enlarged right supraclavicular lymph node. He had non-specific fever, loss of appetite and weight loss. No other positive history. On examination he had non tender lymph node in right cervical reigon about $2 \mathrm{~cm}$. The haematological and biochemical parameters were normal and a tuberculosis skin test for M. tuberculosis showed $7 \mathrm{~mm}$, induration. Chest $X$ ray was normal. Serum samples were positive for antibodies against HIV. CD4+ cell count was 140 /MicroL. FNAC of supraclavicular lymph node showed chronic granulomatous inflammation consistent with tuberculosis. The smear for acid fast bacilli of the specimen were negative. For confirmation of diagnosis, pus was also sent for culture and sensitivity for tuberculosis by bactec method. Patient was put on anti-tubercular treatment (ATT) under DOTS, CAT-I. Pus aspirated from the cold abscess in the neck grew $M$. tuberculosis complex, resistant to Streptomycin, Rifampicin and Isoniazid. During this three month period till the availability of culture and sensitivity report, there was no significant improvement in lymph node size. His ATT regimen was modified according to sensitivity report to Kanamycin, Ethionamide, Ethambutol, Pyrazinamide, Levofloxacin, PAS powder and cycloseriene. He was put on antiretroviral treatment (Lamivudine + stvuvudine + efavirenz). His symptoms improved after starting second line drugs. Clinical improvement occurred after 6 weeks of second line ATT.

\section{Discussion}

Tuberculosis (TB) is an infectious disease with varied clinical presentations, extra-pulmonary forms being more common in HIV patients [6]. High mortality rates have been reported for patients co-infected with extensively drug-resistant tuberculosis (XDR-TB) and HIV, but treatment outcomes have not been reported. Without adequate second-line TB and HIV treatment, reported mortality rates for persons co-infected with XDRTB and HIV approach 100\% [9]. Data regarding treatment of extrapulmonary drug-resistant TB are limited. A few cases are described within larger series of MDR-TB cases. Patients with extrapulmonary TB are at risk of treatment failure due to poor drug penetration to the affected tissue and the lack of accessibility of tissue for serial cultures. Surgical resection (scrofula) and drainage (empyema, abscesses, and arthritis) may decrease bacterial burden and improve outcome. Full medical treatment is still indicated. Drug-resistant TB meningitis is challenging to treat due to the incomplete CSF penetration of many second-line drugs. Intrathecal administration of medications and the use of newer fluoroquinolones may improve outcome and should be evaluated prospectively. Among XDR TB patients who survive to initiation of secondline TB therapy, early treatment outcomes reported describe low rates of sputum culture conversion, major adverse events, and a substantial number of early deaths [10]. Tubercular lymphadenitis involving cervical region is the most common form. Primary multi-drug resistant extrapulmonary tuberculosis is an uncommon form of the disease, but it seems that by increasing the number drug resistant tuberculosis around the world, the number of cases of primary multi-drug resistant tuberculosis with extrapulmonary presentation also is going to rise. By increasing the number of drug resistant tuberculosis patients around the world, appropriate diagnosis and treatment of different presentations of the disease need a special attention [1]. The diagnosis is very difficult requiring high index of suspicion. Definitive diagnosis is made by demonstration of Mycobacterium tuberculosis in tissues or fluids. There is scanty information regarding extra-pulmonary primary drug resistant TB in the literature [7].

Drug resistant tuberculosis has become major public health problem since early 1990 [4]. The prevalence of primary multi drug resistant tuberculosis in India was estimated around $2-3 \%$; however, this prevalence is $15-50 \%$ among previously treated cases [3]. These cases highlight the observation that even extra-pulmonary primary multidrug-resistant tuberculosis can be successfully treated with currently available second line drugs [5].

The four different categories of drug resistant TB are monoresistance, poly-resistance, multidrug-resistance TB (MDR-TB) and extensive drug-resistance TB (XDR-TB). MDR-TB is defined as resistance to at least rifampicin and isoniazid and XDR-TB is defined as MDR-TB plus resistance to any fluoroquinolone, and at least one of three injectable second-line drugs (capreomycin, kanamycin and amikacin). Extra-pulmonary TB among the immunocompetent individuals constitutes about $15 \%$ to $20 \%$ of all cases of TB, and in HIV patients it accounts for more than 50\% [8]. Multidrug-resistant (MDR) and extensively drug-resistant (XDR) tuberculosis are generally thought to have high mortality rates. As, drug resistance is a man made problem, its emergence and spread can be prevented by prompt diagnosis and effective treatment of all drug susceptible and resistant cases [5]. However, many cases can be treated with the right combination and rational use of available antituberculosis drugs. The recommended regimen is the combination of at least four drugs to which the Mycobacterium tuberculosis isolate is likely to be susceptible. 
Drugs are chosen with a stepwise selection process through five groups on the basis of efficacy, safety, and cost. Among the first group (the oral first-line drugs) high-dose isoniazid, pyrazinamide, and ethambutol are thought of as an adjunct for the treatment of MDR and XDR tuberculosis. The second group is the fluoroquinolones, of which the first choice is highdose levofloxacin. The third group are the injectable drugs, which should be used in the following order: capreomycin, kanamycin, then amikacin. The fourth group are called the second-line drugs and should be used in the following order: thioamides, cycloserine, then aminosalicylic acid. The fifth group includes drugs that are not very effective or for which there are sparse clinical data. Drugs in group five should be used in the following order: clofazimine, amoxicillin with clavulanate, linezolid, carbapenems, thioacetazone, then clarithromycin.

High failure rates are usually seen in the treatment of drug resistant TB compared with drug susceptible ones [6]. The Treatment as suggested by CDC is given in the (Figure 1).

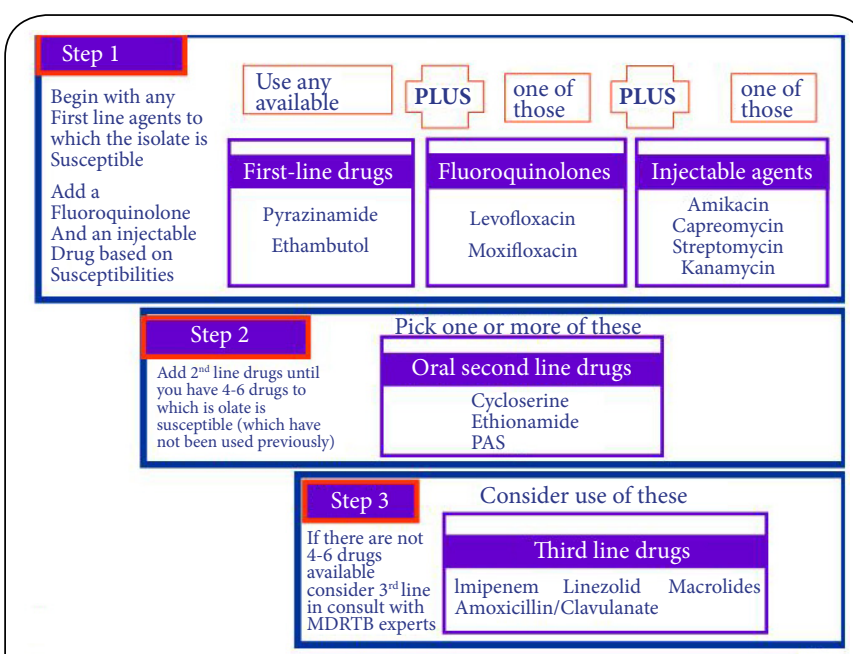

Figure 1. Treatment suggested by CDC.

\section{Critical discussion of literature}

Multidrug resistant tuberculosis has emerged as a significant global health concern. There are alarming reports of increasing drug resistance globally which potentially threaten to disrupt the gains achieved in tuberculosis in last decade. MDR TB is essentially a man made disease due to inadequate treatment. Many cases can be treated with the right combination and rational use of available antituberculosis drugs. Without adequate second-line TB and HIV treatment, reported mortality rates for persons co-infected with extensive XDR TB and HIV approach 100\% [9]. Extrapulmonary MDR-TB is treated with the same strategy involving the same regimen and duration as pulmonary MDR-TB. The second-line drugs are generally widely distributed in most body fluids and tissues [11]. Penetration into cerebrospinal fluid is good with pyrazinamide, ethionamide, prothionamide, cycloserine and newer generations of fluoroquinolones. Aminoglycosides have good penetration in the presence of the meningeal inflammation. Monitoring strategy of the patient is less straightforward and mostly depends on the clinical and radiological observation. Prognosis is relatively better than pulmonary MDR-TB as most of the reported patients with extrapulmonary MDR-TB completed treatment and survived [3].

The prevalence of MDR-TB is increasing in number in recent years because of increased awareness of the disease, increased access to culture and drug sensitivity testing, and earlier suspicion of MDR-TB cases among previously treated patients. So, it is expected that MDR-TB affecting extrapulmonary sites will increase in the near future. Hence, there is an urgent need for increasing awareness of the physicians to such presentation [3].

\section{Competing interests}

The authors declare that they have no competing interests.

Authors' contributions

\begin{tabular}{|l|c|c|}
\hline Authors' contributions & NM & PB \\
\hline Research concept and design & $\checkmark$ & -- \\
\hline Collection and/or assembly of data & $\checkmark$ & -- \\
\hline Data analysis and interpretation & $\checkmark$ & -- \\
\hline Writing the article & -- & $\checkmark$ \\
\hline Critical revision of the article & -- & $\checkmark$ \\
\hline Final approval of article & $\checkmark$ & -- \\
\hline Statistical analysis & $\checkmark$ & -- \\
\hline
\end{tabular}

Publication history

Editor: Massimo De Martinis, University of L'Aquila, Italy. EIC: Fabio Angeli, University of Perugia, Italy. Received: 16-Apr-2014 Final Revised: 01-Aug-2014 Accepted: 07-Aug-2014 Published: 27-Aug-2014

\section{References}

1. Mirsaeidi SM, Tabarsi P, Edrissian MO, Amiri M, Farnia P, Mansouri SD, Masjedi MR and Velayati AA. Primary multi-drug resistant tuberculosis presented as lymphadenitis in a patient without HIV infection. Monaldi Arch Chest Dis. 2004; 61:244-7. I PubMed

2. Mukherjee $S$ and Sarkar S. Treating tuberculous lymphadenitis--ifs and buts. J Indian Med Assoc. 2003; 101:16-7, 23. I PubMed

3. Rawat J, Sindhwani G and Dua R. Primary multi-drug resistant tubercular Iymphadenitis in an HIV infected patient. Indian J Tuberc. 2009; 56:1579. | Pdf | PubMed

4. Pablos-Mendez A, Raviglione MC, Laszlo A, Binkin N, Rieder HL, Bustreo F, Cohn DL, Lambregts-van Weezenbeek CS, Kim SJ, Chaulet P and Nunn P. Global surveillance for antituberculosis-drug resistance, 1994-1997. World Health Organization-International Union against Tuberculosis and Lung Disease Working Group on Anti-Tuberculosis Drug Resistance Surveillance. N Engl J Med. 1998; 338:1641-9. | Article | PubMed

5. Kant $S$, Saheer $S$, Hassan $G$ and Parengal J. Extra-pulmonary primary multidrug-resistant tubercular lymphadenitis in an HIV negative patient. BMJ Case Rep. 2012; 2012. | Article I PubMed Abstract | PubMed Full Text

6. Golden MP and Vikram HR. Extrapulmonary tuberculosis: an overview. Am Fam Physician. 2005; 72:1761-8. I Article I PubMed

7. Tageja N and Sharma V. Multidrug-resistant tuberculosis in India. Lancet. 
Mittal et al. Internal Medicine Inside 2014,

2010; 376:682-3 . | Article | PubMed

8. Sharma SK, Mohan A, Gupta R, Kumar A, Gupta AK, Singhal VK and Pande JN. Clinical presentation of tuberculosis in patients with AIDS: an Indian experience. Indian J Chest Dis Allied Sci. 1997; 39:213-20. | PubMed

9. Gandhi NR, Moll A, Sturm AW, Pawinski R, Govender T, Lalloo U, Zeller $\mathrm{K}$, Andrews $\mathrm{J}$ and Friedland $\mathrm{G}$. Extensively drug-resistant tuberculosis as a cause of death in patients co-infected with tuberculosis and HIV in a rural area of South Africa. Lancet. 2006; 368:1575-80. | Article | PubMed

10. Max R. O'Donnell, Nesri Padayatchi, Charlotte Kvasnovsky, Lise Werner and Iqbal Master C. Robert Horsburgh Treatment Outcomes for Extensively Drug-resistant Tuberculosis and HIV Co-infection. Emerging Infectious Diseases. 2013;19.

11. Guidelines for the programmatic management of drug-resistant tuberculosis. Geneva, World Health Organization, Emergency Update, 2008. | Pdf

\section{Citation:}

Mittal $\mathrm{N}$ and Bansal P. Multidrug resistant extrapulmonary tuberculosis - three case reports and review of literature. Intern Med Inside. 2014; 2:2. http://dx.doi.org/10.7243/2052-6954-2-2 